\title{
Study on the Changes of Aster Tataricus Main Chemical Compositions Before and After Honey-Roasting
}

\author{
Liu Haijun ${ }^{\mathrm{a}}$, Zhang Yaping ${ }^{\mathrm{b}}$, Guo Kailin ${ }^{\mathrm{c}}$, Liu Keyue $\mathrm{d}^{\mathrm{d}^{*}}$ \\ Jiujiang University, Jiangxi, China \\ aemail:392507102@qq.com, bemail:24251958@qq.com, email:gkl123@126.com, \\ $\mathrm{d}^{*}$ email:liukeyue2011@126.com
}

Keywords: Aster tataricus Linn; Honey-roasted; Total triterpenoids; Total flavonoids

\begin{abstract}
Objective: To study the changes of the traditional Chinese medicine Aster tataricus Linn's main chemical compositions before and after honey-roasted, and analyze the main chemical compositions of its two different processed products which includes crude or Honey-roasted. Methods: Using ultraviolet spectrophotometry to measure the content of total triterpenoids and total flavonoids in crude and honey-roasted Aster tataricus L.; The Thin Layer Chromatography (TLC) was employed to compare the changes of petroleum ether, acetic ether and n-butanol between the crude Aster tataricus and the one processed with honey. Results: The amount of flavonoids and total triterpenoids in Aster tataricus L after honey-roasted are significantly higher than those in the crude ones. The TLC showed that flavonoids ingredients significantly increased. Conclusion: The reason why honey-roasted Aster tataricus L. can enhance cough-expectoranting curative effect is due to its change of main effective component, including the increased dissolution of total flavonoids and total triterpenoids, and the synergy of refined honey.
\end{abstract}

\section{Introduction}

The Aster tataricus is a member of the Compositae family. Its root and rhizome have been used as a common traditional Chinese medicine for moistening lungs, depressing qi, dissolving phlegm and relieving a cough ${ }^{[1-2]}$. Aster tataricus L. processed with honey has been recorded in Leigong Treatise on the Preparation in the northern and southern dynasties. It is an important drug in many Chinese herbal medicine compound preparations and is used a lot in clinical prescription, but the mechanism is not clear. The modern pharmacological studies have showed that the honey-roasted Aster tataricus L. could significantly reduce the mice cough and increase the excretory amount of phenol red from its weasand ${ }^{[3,4]}$, and the contents of shionone and epifriedelanol among which are likely to be the major active ingredients to relieve cough and eliminate phlegm. Chen Lin et al ${ }^{[4]}$ adopted high performance liquid chromatography (HPLC) to measure the amount of shionone in the crude and other four kinds of processed Aster tataricus L., and dispalyed that the contents of shionone were increased in crude and honey-roasted Aster tataricus L. A variety of compounds has been isolated from Aster tataricus L. including triterpene and flavonoids ${ }^{[5,6]}$. Then it demonstrates that the improved efficacy after Chinese herbal medicine processing may be caused by the change of chemical compositions. In this study, the modern pharmaceutical analysis method was employed to quantitative and qualitative analysis of the changes in the levels of main chemical compositions in Aster tataricus L. before and after honey-roasted, and explored the correlation between the changes and the efficacy in order to provide a theoretical basis for further illustrating the scientificity of processed Aster tataricus L. with honey and the standardization of the processing technology so as to establish the experimental basis for the further study of exploring the mechanism of honey-roasted Aster tataricus L. 


\section{Organization of the Text}

\section{Materials and Equipment}

Experimental Materials and Reagents. Honey (Shanghai Guanshengyuan Co.,Ltd ); medicine astern (Shanghai Leiyunshang Co., Ltd); reference substance of shionone (National Institute for the Control of Pharmaceutical and Biological Products); reference substance of rutin (National Institute for the Control of Pharmaceutical and Biological Products); sodium nitrite (Shantou Guanghua Chemical Factory); sodium hydroxide (Tianjin Kemiou Chemical Reagent Co., Ltd); aluminium nitrate (Guangdong Taishan Chemical Factory); petroleum ether, n-butanol and ethyl acetate (Tianjin Beike Chemical Reagent Co., Ltd).

The Main Equipment. Ultraviolet and visible spectrophotometer (TU-1901 type, Beijing Purkinjie General Instrument Co., Ltd); rotary evaporator (RE- 52AA type, Shanghai Yarong Biochemical Instrument Factory); circulating vacuum pump (SHZ-IIID type, Shanghai Yarong Biochemical Instrument Factory); electronic analytical balance (BT-224S type, Beijing Sartorius Scientific Instruments Co., Ltd); digital thermostat water bath (WB-5100 type, Tianjin Aotesaisi instrument Co.,Ltd);

\section{Experimental Method}

TLC Comparison of Different Polarity Sites Between Crude and Honey-roasted Aster Tataricus $\boldsymbol{L}$. The crude and honey-roasted powder of Aster tataricus $L$. were reflux extracted 2 times with $85 \%$ ethanol, respectively, and each time for 4 hours; then the extracts were evaporated under reduced pressure to recover the solvent, and were washed successively by petroleum ether, ethyl acetate and n-butanol. $10 \mu \mathrm{L}$ extract liquor was spotted on the thin layer plate to proceed TLC, with refined honey diluent as the reference substance to explore the triterpenoids, flavonoids and saponins.

Extraction and Content Determination of Total Triterpenoids in Crude and Honey-roasted Aster Tataricus L. The reference substance of shionone was prepared in ethyl acetate at the concentration of $0.1 \mathrm{mg} \cdot \mathrm{ml}^{-1}$. Measure reference solution $0.1,0.2,0.4,0.6,0.8,1.0$ and $1.2 \mathrm{ml}$ respectively to the scale test tubes, and then degrease the solvent by water bath. After cooling, drip and mix $0.2 \mathrm{ml} 5 \%$ vanillin-acetic acid solution and $0.8 \mathrm{ml}$ perchloric acid. Plug the tubes and put them into the $65^{\circ} \mathrm{C}$ water bath to heat 20 minutes; after the release of ice-water bath, add $5 \mathrm{ml}$ glacial acetic acid and shake stand at room temperature. Finally, measure the absorbance values in which the absorbance as the vertical axis, and the shionone concentration as horizontal axis, the regression equation is that $\mathrm{Y}$ $=0.007 \mathrm{X}+0.019, \mathrm{r}=0.9993$.

Combine the $250 \mathrm{~g}$ crude and honey-roasted powder of aster tataricus L. respectively with $85 \%$ ethanol under reflux extraction twice and each time for 4 hours; then the combined extracts are evaporated under reduced pressure to recover the solvent, and the extracts are washed with acetic ether to explore the content of triterpenoid in the extract of crude and honey-roasted Aster tataricus L.with refined honey diluent as the reference substance.

Extraction and Content Determination of Total Flavonoids in Crude and Honey-roasted Aster Tataricus L. Dissolve $5.0 \mathrm{mg}$ reference substance of rutin in defined amount of $75 \%$ ethanol to $50 \mathrm{ml}$, and mix $100 \mu \mathrm{g} \cdot \mathrm{ml}^{-1}$ rutin standard stock solution. Drip $1 \mathrm{ml}, 2 \mathrm{ml}, 3 \mathrm{ml}, 4 \mathrm{ml}$ and $5 \mathrm{ml}$ standard stock solution respectively to the $10 \mathrm{ml}$ tubes, dissolve $75 \%$ ethanol to $5 \mathrm{ml}$; then add $0.3 \mathrm{ml} 5 \%$ $\mathrm{NaNO}_{2}$ solution and place for $6 \mathrm{~min}$; readd $0.3 \mathrm{ml} 10 \% \mathrm{Al}\left(\mathrm{NO}_{3}\right)_{3}$ solution and place for $6 \mathrm{~min}$; finally drip $4 \mathrm{ml} 1 \mathrm{~mol} \cdot \mathrm{L}^{-1} \mathrm{NaOH}$ solution, dissolve $75 \%$ ethanol to $10 \mathrm{ml}$ and place for $15 \mathrm{~min}$ to measure the absorbance value. The regression equation is that $\mathrm{Y}=8.88669 \mathrm{X}+0.00147, \mathrm{R}^{2}=0.9997$.

Combine the $250 \mathrm{~g}$ crude and honey-roasted powder of Aster tataricus L. respectively with $500 \mathrm{~g}$ $85 \%$ ethanol under reflux extraction twice and each time for 4 hours; then the combined extracts are evaporated by using rotary evaporators to recover the ethanol to explore the total flavonoid content in 
the extract of crude and honey-roasted Aster tataricus L.with refined honey diluent as the reference substance.

\section{Experimental Results}

\section{TLC Comparison in Different Polarity Sites Between Crude and Honey-roasted Aster Tataricus} L.

TLC Comparison in Petroleum ether. Chromatographic conditions: petroleum ether - ethyl acetate (7: 1), the chromogenic reagent is $10 \%$ sulfuric acid in ethanol. The results show that the compositions in honey-roasted aster tataricus L. have significant changes: some of the spots disappear. The specific result is showed in figure 1, in which the object from left to right in turn is 1. refined honey; 2. shionone; 3 . crude aster tataricus L. and 4. honey-roasted aster tataricus L.

TLC Comparison in Ethyl Acetate. Chromatographic conditions: butanone - ethyl acetate methanol - water (5: 3: 3: 1); observing conditions: under $365 \mathrm{~nm}$ ultraviolet fluorescent lamp; the results show that the compositions in honey-roasted aster tataricus L. have significant changes: the spots deepened. The preliminary analysis is that the flavonoid in refined honey is more likely to dissolution or the refined honey make the flavonoid in aster tataricus L. easier to dissolution. The specific result is showed in figure 1 , in which the object from left to right in turn is 1 . refined honey; 2 . crude aster tataricus L. and 3. honey-roasted aster tataricus L.

TLC Comparison in N-butanol. Chromatographic conditions: butanol - ethyl acetate - water (4: 1: 5 upper), the chromogenic reagent is $10 \%$ sulfuric acid in ethanol. The results show that the compositions in honey-roasted aster tataricus L. have significant changes: a slight change in saponins, spots weakened, while many flavonoids detected. A preliminary analysis is that the flavonoid in refined honey is more likely to dissolution or the refined honey make the flavonoid in aster tataricus $\mathrm{L}$. easier to dissolution. The specific result is showed in figure 1, in which the object from left to right in turn is 1 . refined honey; 2 . crude aster tataricus L. and 3. honey-roasted aster tataricus L.
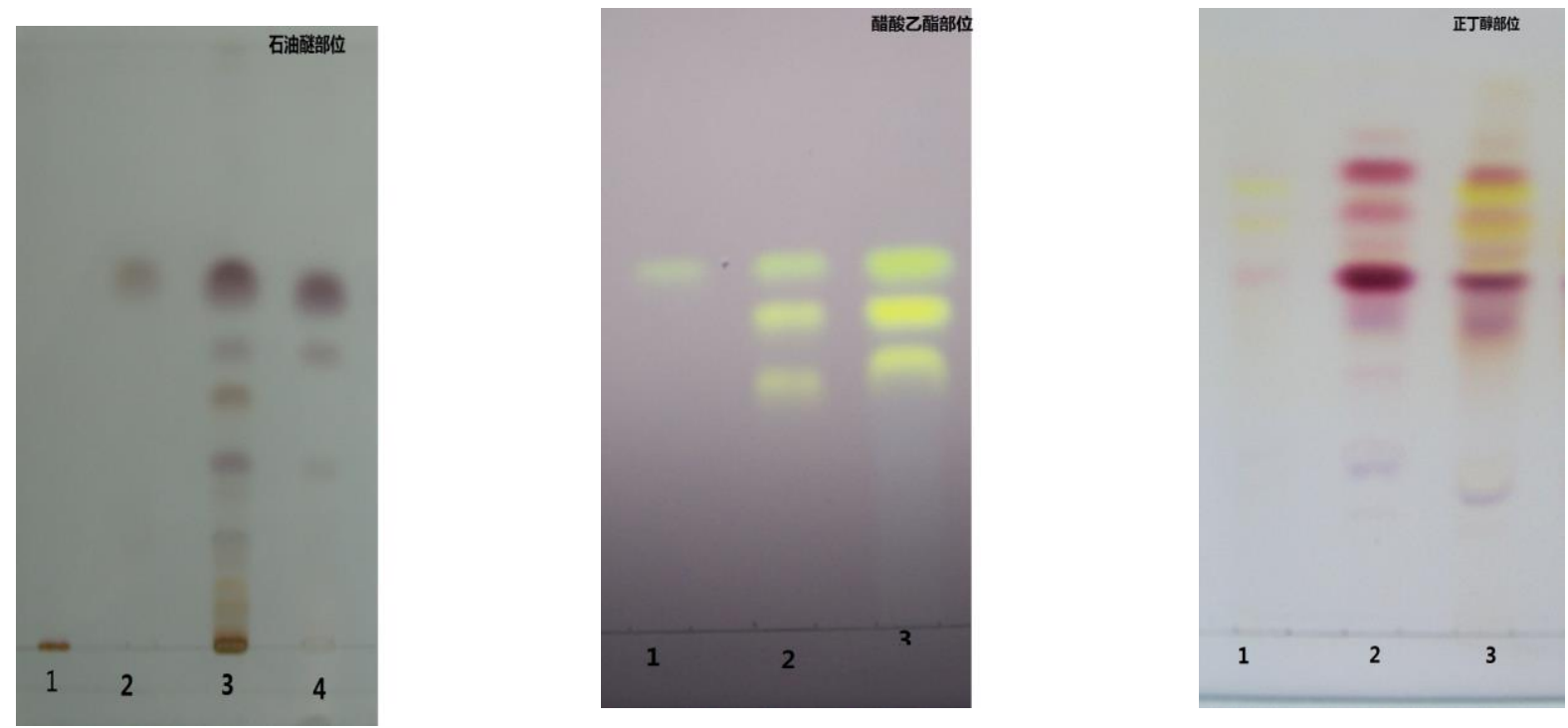

Figure. 1 The thin layer contrast diagram of crude and honey-roasted Aster tataricus L.'s petroleum ether, Ethyl acetate, and n-butanol extract parts

Comparison in the Total Triterpenoids Content Between Crude and Honey-roasted Aster Tataricus L.

Vanillin - perchloric acid method is adopted to conduct a comparative study of the total triterpenoids content between crude and honey-roasted aster tataricus L. The study found that total triterpenoid content is $1.992 \%$ in honey-roasted aster tataricus L., while the total triterpenoid content is 
$1.669 \%$ in crude aster tataricus L., which indicates that the total triterpenoid content has significantly increased after honey-roasting, and its efficiency are positively correlated.

\section{Comparison in the Total Flavonoids Content Between Crude and Honey-roasted Aster Tataricus L.}

Sodium nitrite -aluminum nitrate method is adopted to conduct a comparative study of the total flavonoid content between crude and honey-roasted aster tataricus L. The study found that total flavonoid content is $8.351 \%$ in honey-roasted aster tataricus L., while the total flavonoid content is $4.975 \%$ in crude aster tataricus L., which indicates that the total flavonoid content has significantly increased after honey-roasting, and its efficiency are positively correlated.

\section{Conclusion and Discussion}

Comparative studies are conducted to research the changes of aster tataricus L.' chemical compositions before and after processing with honey, which discover that some medicinal active ingredients' content of honey-roasted aster tataricus Linn is higher than the crude one. Following aspects are included: the total saponins and flavonoids content increase, and show a positive correlation with efficiency; the TLC shows that the total flavonoids content increase, and show a positive correlation with efficiency.

It follows that the medicinal compositions undergo quantitative and qualitative changes in the honey-roasted aster tataricus L. The efficacy improved may be caused by the increase of content, or the superposition of refined honey's efficacy of moistening lung to arrest cough. The correlation between the pharmacological efficacy trials and compositions needs to be further study.

\section{Fund Program:}

Science and Technology Plan of Jiangxi Province Education Department (No. GJJ14740)

\section{References}

[1] Hou Haiyan, Chen Li, Dong Junxing. Advance on chemical constituents and pharmacological activities of aster tataricus ,J. Chinese Pharmaceutical Journal. 41(2006): 161-163

[2] .Gong Qianfeng. Science of processing of traditional Chinese Medicine, China press of traditional Chinese medicine,Bei Jin,2003.

[3] Lu Yanhua, Dai Ji, Wang Zhengtao, et al. The efficacy of expectorant and antitussive of aster tataricus and its effective parts and compositions,J. Chinese Herbal Medicine. 30(1999): 360-362.

[4] Zhou Rigui, Tu Jianxiong. Antitussive effects to mice of the processing aster tataricus,J. Hunan

Guiding Journal of Traditional Chinese Medicine and Pharmacology. 6(2000): 56-57.

[4] Xiu Yanfeng, Cheng Xuemei, Liu Lei. Comparison of shionone content in different slices of prepared radix aster ,J. Journal of Shanghai University of Chinese Medicine. 20(2006): 59-61

[5] Liu Keyue, Zhang Tiejun, Zhang Wenyuan, et al.. Triterpenes and steroids from aster tataricus ,J. Natural Product Research and Development. 18(2006): 4-6.

[6] Liu Keyue, Zhang Tiejun, Gao Wenyuan, et al. Phenolic compounds isolated form root and rhizoma of aster tataricus L ,J. Chinese Herbal Medicine. 12(2007): 1793-1795.

[8] Lu Fei,Ren Xiaoqian,Zhang Zhaofeng, et al. Studies on the chemical constituents of extract n-butanol aster, J.Asia Pacific Traditional Medicine.8(2013):42-43 
[9]Yang Bin, Xiao Yongqing,Liang Rixin, et al. Study on the chemical constituents of volatile oil in Radix Asteris expectorant, J. Chinese Journal of Chinese.3(2008):281-283

[10] Zhang Jia-li ,Jin Xing,Wang Hongwu. Analysis of volatile oil of Flos farfarae medicine, aster and its single herb in the GC-MS, J. Fine chemical .3(2012):254-257 